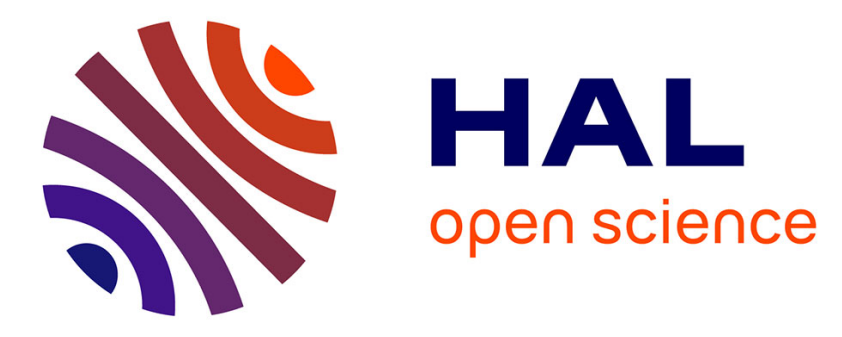

\title{
Numerical analysis of unsteady cavitating flow in an axial inducer
}

Rafael Campos-Amezcua, Farid Bakir, Alfonso Campos-Amezcua, Sofiane Khelladi, Manuel Palacios-Gallegos, Robert Rey

\section{To cite this version:}

Rafael Campos-Amezcua, Farid Bakir, Alfonso Campos-Amezcua, Sofiane Khelladi, Manuel PalaciosGallegos, et al.. Numerical analysis of unsteady cavitating flow in an axial inducer. Applied Thermal Engineering, 2015, 75, pp.1302-1310. 10.1016/j.applthermaleng.2014.07.063 . hal-02444681

\section{HAL Id: hal-02444681 \\ https://hal.science/hal-02444681}

Submitted on 18 Jan 2020

HAL is a multi-disciplinary open access archive for the deposit and dissemination of scientific research documents, whether they are published or not. The documents may come from teaching and research institutions in France or abroad, or from public or private research centers.
L'archive ouverte pluridisciplinaire HAL, est destinée au dépôt et à la diffusion de documents scientifiques de niveau recherche, publiés ou non, émanant des établissements d'enseignement et de recherche français ou étrangers, des laboratoires publics ou privés. 


\title{
Numerical analysis of unsteady cavitating flow in an axial inducer
}

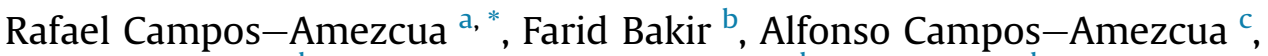 \\ Sofiane Khelladi ${ }^{\text {b }}$, Manuel Palacios-Gallegos ${ }^{\mathrm{d}}$, Robert Rey ${ }^{\mathrm{b}}$ \\ a Instituto de Energías Renovables, Universidad Nacional Autónoma de México, Priv. Xochicalco s/n, Col. Centro, 62580 Temixco, Mexico \\ b DynFluid Laboratory, Arts et Metiers ParisTech, 151 Boulevard de l'Hopital, 75013 Paris, France \\ ${ }^{\mathrm{c}}$ Department of Turbomachinery, Electric Research Institute, 113 Reforma Avenue, Col. Palmira, 62490 Cuernavaca, Mexico \\ ${ }^{\mathrm{d}}$ Research Group of Energy and Sustainability, Chiapas Polytechnic University, Eduardo J. Selvas s/n, Col. Magisterial, 29082 Tuxtla Gutierrez, Mexico
}

\begin{abstract}
A B S T R A C T
This work presents the results of numerical simulation of unsteady cavitating flow through a two -bladed axial inducer. First, the analysis was carried out in a blade cascade, this two-dimensional simplified model, obtained from the studied axial inducer, was used as a test case. Later, the numerical simulations were extended to the original three-dimensional inducer. All numerical calculations were realized in cavitating flow regime. Initially, the results were obtained in steady state, and then in unsteady state.

The main purpose of this study is to explore the local cavitation instabilities, such as alternate blade cavitation and rotating blade cavitation, which can appear in this type of devices when they work under certain operating conditions.

The numerical results show that the fluid flow in the axial inducer is altered by the emergence of the cavitation. These vapor regions are formed, firstly near to the leading edge of each blade. The behavior of the cavitation depends on the operating conditions of the inducer, mainly by the flow rate and the suction pressure.

The numerical simulation was performed using a commercial code based on a cell-centered finite -volume method. The cavitation model used for calculations assumes a thermal equilibrium between phases. It is based on the classical conservation equations of the vapor phase and a mixture phase, with mass transfer due to the cavitation appearing as a source and a sink term in the vapor mass fraction equation. The mass transfer rate is derived from a simplified Rayleigh-Plesset model for bubble dynamics.
\end{abstract}

\section{Introduction}

An inducer is a key component of rocket engine pumps, which improves suction performance. Generally, the inducers have few blades, and the length of them is longer than those of standard axial pump impellers. This singular geometry allows them to operate under very low suction pressure conditions without an important deterioration of pumping performance. If suction pressure of the pump is close to vapor pressure of working fluid, the pump can suffer serious operational difficulties, caused by the cavitation emergence.

The cavitation inception and development on the inducers depend on many parameters: the blade profile, camber, thickness,

\footnotetext{
* Corresponding author

E-mail address: rca@ier.unam.mx (R. Campos-Amezcua).
}

incidence angle and leading edge shape, as well as the walls roughness, the upstream turbulence, vapor pressure, air content, etc. [1].

Many experimental studies have been conducted to understand and avoid the harmful effects of cavitation. Noguera et al. [2] and Bakir et al. [3] have already studied experimentally, in non-cavitating and cavitating regimes, the influence of geometrical parameters such as the shape of the blade leading edge and its sharpening. Tsujimoto et al. [4] introduced for the first time the cross-correlation and phase analysis technique in order to detect the frequency of oscillation and the spatial characteristics of the cavitating flow oscillations. Cervone et al. [5] put in place an hydrodynamic tests bench by means of a high-speed camera, in order to carry out investigations of cavitation flow instabilities on a three-bladed inducer. Analyzes included both inlet pressure signal as well as video acquisitions, which showed the occurrence of a 
cavity length oscillation. Yoshida et al. [6,7] conducted experiments with liquid nitrogen at different temperatures to investigate the influence of the thermodynamic effect on rotating cavitation in an inducer and the relationship between the thermodynamic effect and cavitation instabilities. Franc et al. [8,9] carried out experiments with cold water and refrigerant 114 to determine the thermodynamic delay in the development of leading edge cavities and the thresholds for the onset of cavitation instabilities.

Computational Fluid Dynamics (CFD) has been widely used to simulate non-cavitating flows in turbomachines. In the last decade, CFD has also been accepted to predict cavitating flows in inducers thanks to the remarkable progresses that have been implemented in the cavitation models. The combination of numerical approaches and experimental techniques has turned out to be a powerful tool in the investigation of cavitating flows. Bakir et al. [10] presented numerical and experimental investigations of the cavitating behavior of an axial inducer. Mejri et al. [11] performed a comparison between experimental and simulated results on the overall performances, cavity sizes and cavity location of three industrial inducers, in where a qualitative agreement between experimental and predicted results was found for two inducers for a range of flow rates. Campos-Amezcua et al. [12,13] carried out three-dimensional numerical simulations and experimental investigations of the cavitating flow through an axial inducer to determine the influence of radial tip clearance on inducer overall performance and cavitation behavior.

Many investigations concerning the cavitating flow have used numerical simulations because CFD provides acceptable approaches and due to difficulties to carry out laboratory tests. Tani et al. [14] investigated the relationship between rotating cavitation and flow coefficient using a CFD code. They observed that the negative flow divergence caused by cavity collapse has a great influence on the flow angle, which induces backflow from the tip clearance, and the cavitation instability. Ji et al. $[15,16]$ used numerical simulations based on the Reynolds-Averaged Navier-Stokes (RANS) equations and Large Eddy Simulation (LES) coupled with a mass transfer cavitation model to predict the evolution of the unsteady cavitation and the pressure fluctuations around a conventional marine propeller and a twisted hydrofoil, respectively. Coutier-Delgosha et al. [17] and Goncalves et al. [18,19] conducted numerical simulations solving RANS equations, associated with a barotropic vapor/liquid state law and a simple equation of state, respectively; to simulate turbulence effects on cavitating flows. Numerical results of unsteady cavitating flows in a venturi-type section were compared with experimental data verifying that the turbulence modeling plays a major role in the capture of unsteady behaviors.

This paper presents numerical results of unsteady cavitating flow in a two-bladed axial inducer. Calculations were performed using the commercial code Fluent V6.3.26. The work begins with a brief description of the physical models and numerical aspects used for numerical calculations. After that, numerical results are presented; firstly, for a blade cascade, where the results show flow instabilities, which were observed at partial flow rates, and low suction pressures. The instabilities observed in the simplified model were the alternate blade cavitation and the rotating blade cavitation. Finally, the numerical analysis was extended to a three-dimensional inducer model, where the rotating blade cavitation was also observed under certain operating conditions.

\section{Numerical method and physical model}

The non-cavitating and cavitating flow through an axial inducer was modeled for two flow rates, to different cavitation conditions. The geometry of inducer studied in this work is shown in Fig. 1, and its main characteristics are listed in Table 1.
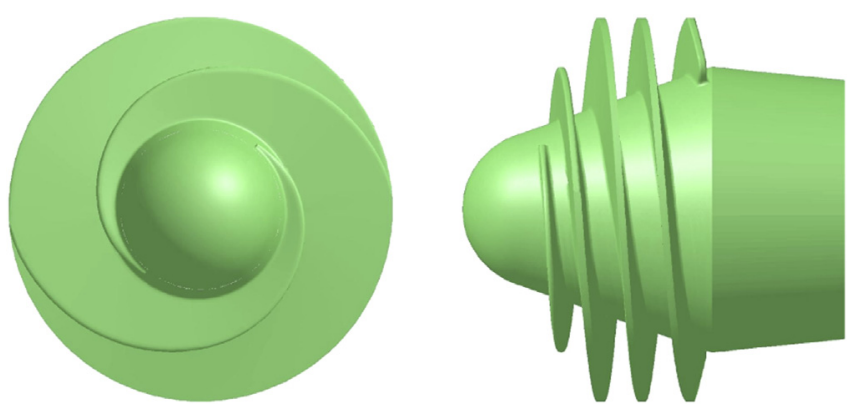

Fig. 1. Two-bladed axial inducer.

Numerical simulations were carried out using as working fluid water at $300 \mathrm{~K}$. The densities of liquid water and water vapor are $\rho_{l}=1,000 \mathrm{~kg} / \mathrm{m}^{3}$ and $\rho_{v}=0.5542 \mathrm{~kg} / \mathrm{m}^{3}$, respectively. Saturation pressure is $p_{\text {sat }}=3,540 \mathrm{~Pa}$ and surface tension is $\sigma_{s}=0.0717 \mathrm{~N} / \mathrm{m}$. The non condensable gas mass fraction was defined as $\gamma_{g}=15 \mathrm{ppm}$ based on [20] and validated for a steady cavitating regime [21].

The first results were obtained using the RNG $\kappa-\varepsilon$ turbulence model. Later, calculations were carried out using RNG $\kappa-\varepsilon$ modified model to take into account both intrinsic and system instabilities. In both cases, standard wall function was used as near-wall treatment. The choice of these two turbulence models was based on the works of Coutier-Delgosha et al. [17] and Goncalves et al. [18,19].

\subsection{Numerical method}

The commercial code used for all simulations was Fluent 6.3.26. This code employs a cell-centered finite-volume method that allows the use of computational elements with arbitrary polyhedral shape.

Convective terms are discretized using the second order upwind scheme. The velocity-pressure coupling and overall solution procedure are based on a SIMPLE type segregated algorithm adapted to unstructured grids. The discretized equations are solved using point wise Gauss-Seidel iterations, and an algebraic multi-grid method accelerates the solution convergence. The convergence criteria in the present numerical analysis were at least of three orders of magnitude drop in the mass conservation imbalance and momentum equation residuals, which are deemed sufficient for most steady flow solutions. A more detailed description of the numerical method is available in Ref. [22].

\subsection{Cavitation model}

The cavitation model used for this study was developed by Singhal et al. [20]. It takes into account all first order effects. The influence of slip velocity between the liquid and the vapor phases was not considering.

Table 1

Main characteristics of inducer.

\begin{tabular}{ll}
\hline Parameter & Value \\
\hline Rotational speed, $\omega$ & $8,000 \mathrm{rpm}$ \\
Maximal efficiency, $\eta_{\max }$ & $15.5 \%$ \\
Nominal flow coefficient, $\Phi_{\text {nom }}$ & 0.014 \\
Nominal head coefficient, $\Psi_{\text {nom }}$ & 0.188 \\
Tip diameter, $D_{\text {tip }}$ & $50 \mathrm{~mm}$ \\
Tip chord length, $l_{\text {blade }}$ & $198 \mathrm{~mm}$ \\
Solidity, $S_{\text {tip }}=l_{\text {blade }} / \mathrm{h}$ & 2.52 \\
Tip clearance, $\delta_{\text {tip }}$ & $0.65 \mathrm{~mm}$ \\
Blades number, $Z$ & 2 \\
Inlet tip blade angle, $\beta_{\text {tip }-1}$ & $4^{\circ}$ \\
\hline
\end{tabular}


For the multi-phase flow solution, the single-fluid mixture model was employed. The mixture model solves the continuity and momentum equation for the mixture, and the volume fraction equation for the secondary phases.

The cavitation model consists in solving the standard incompressible Reynolds-Average Navier-Stokes equations with the use of a conventional turbulence model. The working fluid is assumed to be a mixture of liquid, vapor and non condensable gases.

The mixture density, $\rho$, is defined by:

$\frac{1}{\rho}=\frac{\gamma_{v}}{\rho_{v}}+\frac{\gamma_{g}}{\rho_{g}}+\frac{1-\gamma_{v}-\gamma_{g}}{\rho_{l}}$

with

$\gamma_{v}=\frac{\alpha_{v} \rho_{v}}{\rho}, \quad \gamma_{g}=\frac{\alpha_{g} \rho_{g}}{\rho}$, and $\gamma_{l}=\frac{\alpha_{l} \rho_{l}}{\rho}=1-\gamma_{v}-\gamma_{l}$

where $\alpha_{g}, \alpha_{l}$ and $\alpha_{v}$ are the non condensable gases, liquid and vapor volume fraction, respectively; and $\alpha=\alpha_{g}+\alpha_{v}$ is the total vapor volume fraction.

The vapor mass fraction, $\gamma$, is governed by the transport equation given by:

$\frac{\partial}{\partial t}(\rho \gamma)+\nabla \cdot(\rho \mathbf{v} \gamma)=\nabla \cdot(\Gamma \nabla \gamma)+R_{e}-R_{C}$

where $\mathbf{v}$ is the velocity vector of the vapor phase, $\Gamma$ is the effective exchange coefficient, and $R_{e}$ and $R_{c}$ are the vapor generation and condensation rate terms (or phase change rates). The above formulation employs a homogenous flow approach.

$R_{B} \frac{D^{2} R_{B}}{D t^{2}}+\frac{2}{3}\left(\frac{D R_{B}}{D t}\right)^{2}=\frac{P_{B}-P}{\rho_{l}}-\frac{4 \nu_{l}}{R_{B}} \frac{D R_{B}}{D t}-\frac{2 \sigma_{s}}{\rho_{l} R_{B}}$

Using the Rayleigh-Plesset equation (4), without the viscous damping and surface tension terms and combining with the continuity equations, the expression for the phase change rate is obtained as:

$R=(n 4 \pi)^{1 / 3}(3 \alpha)^{2 / 3} \frac{\rho_{v} \rho_{l}}{\rho}\left[\frac{2}{3}\left(\frac{P_{B}-P}{\rho_{l}}\right)-\frac{2}{3} R_{B} \cdot \frac{D^{2} R_{B}}{D t^{2}}\right]^{1 / 2}$

Employing the above equation and ignoring the second order derivative of $R_{B}$, the simplified equation for vapor transport is obtained as:

$\frac{\partial}{\partial t}(\rho \gamma)+\nabla \cdot(\rho \gamma \mathbf{v})=(n 4 \pi)^{1 / 3}(3 \alpha)^{2 / 3} \frac{\rho_{v} \rho_{l}}{\rho}\left[\frac{2}{3}\left(\frac{P_{B}-P}{\rho_{l}}\right)\right]^{1 / 2}$

The vapor volume fraction can be related to the bubble number and radius of bubble as:

$\alpha_{v}=\frac{4}{3} n \pi R_{B}^{3}$

Turbulent effects are taken into account by:

$P_{v}=P_{\text {sat }}+0.195 \rho \kappa$

The phase change rate expressions are derived from equation (6) as:

$R_{e}=C_{e} \frac{\sqrt{\kappa}}{\sigma_{s}} \rho_{v} \rho_{l}\left[\frac{2}{3} \frac{P_{v}-P}{\rho_{l}}\right]^{1 / 2}\left(1-\gamma_{v}-\gamma_{g}\right)$
$R_{c}=C_{c} \frac{\sqrt{\kappa}}{\sigma_{s}} \rho_{l} \rho_{l}\left[\frac{2}{3} \frac{P-P_{v}}{\rho}\right]^{1 / 2} \gamma_{v}$

where $C_{e}=0.02$ and $C_{c}=0.01$ are empirical coefficients, and $\kappa$ is the local turbulent kinetic energy. A more detailed description of the cavitation model is available in Ref. [20].

\section{3. $R N G K-\varepsilon$ turbulence model}

This model was developed by Yakhot et al. [23]. It uses the Re-Normalization Group (RNG) methods to renormalize the Navier-Stokes equations, and take into account the effects of smaller scales of motion. The RNG $K-\varepsilon$ model is derived from standard $\kappa-\varepsilon$ model. The main difference is the form of the dissipation of the kinetic turbulent energy equation.

The turbulence kinetic energy, $\kappa$, and its rate of dissipation, $\varepsilon$, are obtained from the following transport equations:

$\frac{\partial}{\partial t}(\rho \kappa)+\frac{\partial}{\partial x_{i}}\left(\rho \kappa u_{i}\right)=\frac{\partial}{\partial x_{j}}\left(\alpha_{\kappa} \mu_{\text {eff }} \frac{\partial \kappa}{\partial x_{j}}\right)+G_{\kappa}-\rho \varepsilon$

and

$\frac{\partial}{\partial t}(\rho \varepsilon)+\frac{\partial}{\partial x_{i}}\left(\rho \varepsilon u_{i}\right)=\frac{\partial}{\partial x_{j}}\left(\alpha_{\varepsilon} \mu_{e f f} \frac{\partial \varepsilon}{\partial x_{j}}\right)+C_{1 \varepsilon} \frac{\varepsilon}{\kappa} G_{\kappa}-C_{2 \varepsilon}^{*} \rho \frac{\varepsilon^{2}}{\kappa}$

where $C_{2 \varepsilon}^{*}=C_{2 \varepsilon}+C_{\mu} \eta^{3}\left(1-\eta / \eta_{0}\right) / 1+\beta \eta^{3}, \eta=S \kappa / \varepsilon, S=\left(2 S_{i j} S_{j i}\right)^{1 / 2}$ and $G_{K}$ represents the production of turbulence kinetic energy.

$P_{\kappa}=-\rho \overline{u_{i}^{\prime} u_{j}^{\prime}} \frac{\partial u_{j}}{\partial u_{i}}$

The turbulent viscosity, $\mu_{t}$, is given by:

$\mu_{t}=\rho C_{\mu} \frac{\kappa^{2}}{\varepsilon}$

The constants of the model are: $C_{\mu}=0.0845, C_{1} \varepsilon=1.42$, $C_{2 \varepsilon}=1.68, \alpha_{K}=\alpha \varepsilon=1.393, \eta_{0}=4.38$, and $\beta=0.012$.

\section{4. $R N G K-\varepsilon$ modified turbulence model}

This turbulence model is an adaptation from the RNG $K-\varepsilon$ model and was proposed by Reboud et al. [24]. The modification concerns the reduction, in the low vapor ratio regions, of the effective viscosity, $\mu_{\text {eff }}=\mu_{\text {flow }}+\mu_{t}$. For this, the mixture turbulent viscosity is given by:

$\mu_{t}=f(\rho) C_{\mu} \frac{\kappa^{2}}{\varepsilon}$,

where mixture density function, $f(\rho)$, is given by:

$f(\rho)=\rho_{v}+\left(\frac{\rho_{v}-\rho}{\rho_{v}-\rho_{l}}\right)^{n}\left(\rho_{l}-\rho_{v}\right)$

where $n \gg 1$

Calculations were performed with the recommended exponent value $n=10$ [18], the turbulent viscosity in the region with higher vapor volume fraction is reduced to better simulate the reentrant jet and shedding behavior. The RNG $\kappa-\varepsilon$ modified model was implemented in initial code as a User's Defined Function supplied by Fluent. 


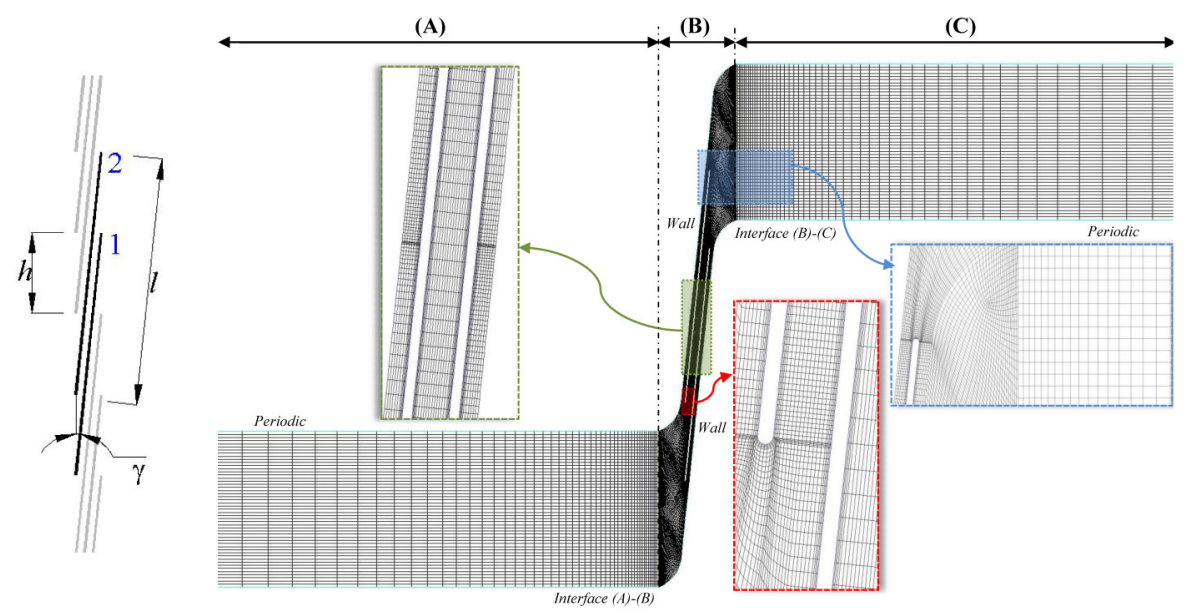

a) Blade cascade

b) Boundary conditions and near-wall mesh resolution

Fig. 2. Blade cascade corresponding to two-bladed axial inducer.

\section{Geometrical model and grid generation}

\subsection{Two-dimensional inducer}

First, the numerical study was carried out on a blade cascade, which was built at $85 \%$ of inducer blade span. In order to impose moving mesh conditions, the numerical domain was divided into three sub-domains, namely: upstream region (A), blade-to-blade region (B), and downstream region (C), see Fig. 2. Tangential velocity $\left(U=\omega \cdot r_{85 \%}\right)$ was imposed in the moving region (B) using a sliding mesh technique, whereas regions (A) and (C) were defined as static regions.

Boundary conditions at the inlet and outlet of control domain were placed distant enough from the leading and trailing edges of blade $\left(l_{\text {upstream }}=l_{\text {downstream }}=15 \cdot l_{\text {blade }}\right)$, in order to avoid its impacts on the calculations.

The following boundary conditions were used to define the control domain:

1. Constant velocity at the inlet, $v_{1}=Q / A_{1}$. The nominal flow, $Q_{\text {nom, }}$ was defined to correspond to an incidence angle of zero.

2. Constant static pressure at the outlet. This boundary condition was modified gradually to get different cavitation conditions.

3. Non-slip condition at the blades boundaries.

4. Sliding interfaces at the limits between (A)-(B) sub-domains and $(B)-(C)$ sub-domains.

5. Translational periodic condition was applied for two successive blades.

The discretization of the calculation domain was done with a rectangle-like structured grid. A grid study was carried out on non cavitating flow. Three different meshes were tested: a coarse mesh $(300 \times 50)$, a fine mesh $(500 \times 50)$, and a refined mesh $(650 \times 50)$.
The first coarse mesh presented backflow problems at outlet, because of a very important aspect ratio of grid in downstream region. Moreover, the fine and refine meshes presented similar results.

Furthermore, three different lengths of inlet and outlet sub-regions were tested: $l_{\text {upstream }}=l_{\text {downstream }}=5 \cdot l_{\text {blade }}, 10 \cdot l_{\text {blade, }}$, and

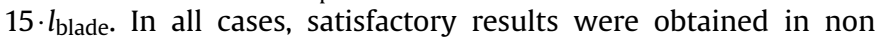
cavitating flow. However, when the cavitating flow was simulated, first and second cases presented backflow problems in the outlet of domain. Problems of divergence were also observed when the outlet boundary was placed close to the blade cascade, mainly for too small values of cavitation number. In conclusion, all calculations were carried out on fine mesh $(500 \times 50)$ with inlet/outlet sub-domain length of $l_{\text {upstream }}=l_{\text {downstream }}=15 \cdot l_{\text {blade }}$.

Boundary layer meshing was used to ensure adequate mesh refinement near the walls and thus a small dimensionless factor $y^{+}$, see Fig. 2. A $1 \mathrm{~mm}$ first cell distance was imposed with a growth rate of 1.2 which allowed values of $y^{+}$between 6 and 51 .

\subsection{Three-dimensional inducer}

A hybrid grid was generated using the preprocessor Gambit, for modeling and simulation of the inducer in three dimensions. The computational domain was divided into four sub-blocks: an upstream region lengthened until $2.6 \cdot D_{\text {tip }}$ from the leading edge, a blade-to-blade region, a downstream region lengthened until $2.4 \cdot D_{\text {tip }}$ from the leading edge, and the tip clearance region formed by a ring of thickness, $\delta_{t}$, and an axial length, $l_{\text {rotor, }}$ see Fig. 3 .

The control domain was discretized using the following methodology: first the blade surfaces were meshed with triangular cells, focusing on tip and blade edges, where smaller cells were used. Later, the blade-to-blade region was filled with tetrahedral type cells. Finally, the rest of blocks were meshed with prism type cells.

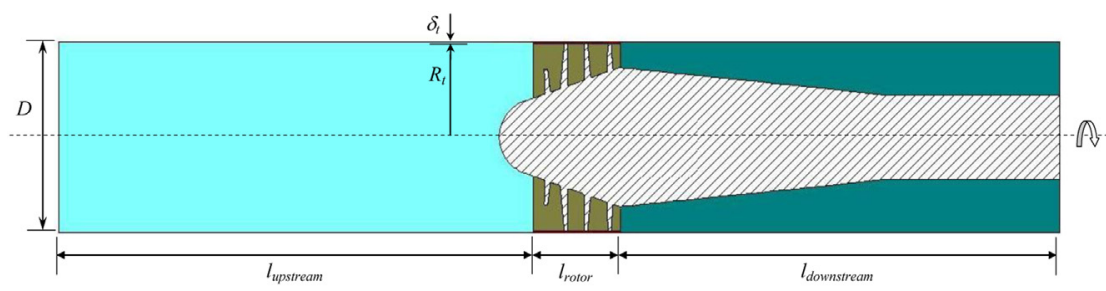

Fig. 3. Sub-blocks of whole computational domain. 

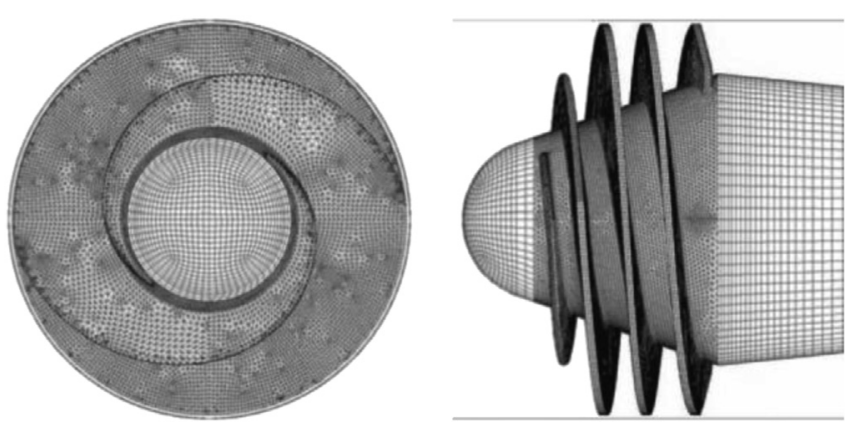

Fig. 4. Computational grid on the inducer walls.

For the grid independence study, four computational grids were generated, following the same meshing strategy. The numbers of cells of these test meshes are: (a) 480,185; (b) 800,154; (c) $1,228,668$; and (d) $1,416,418$. The mesh (c) was retained for all calculations because it presented the best accurate/computational time rate. Fig. 4 shows the front and lateral views of the surfaces grid. Finally, a grid independence study at tip clearance region was performed using, in radial direction, 3, 12, 25 and 30 equidistant prism type cells. The 25 radial-cells grid was selected for the numerical simulations.

Similar to the case of two-dimensional inducer, a constant flow rate was defined at inlet boundary, which was varied from $\Phi=0.0175$ to $\Phi=0.0050$. Moreover, a constant static pressure was defined at the outlet boundary, which was modified gradually to get different cavitation conditions. Conformal grid interfaces were used in the boundaries of the regions "rotor-clearance", and non-conformal grid interfaces were used in the boundaries of the regions "upstream-rotor" and "rotor-downstream".

\section{Numerical results}

\subsection{Two-dimensional inducer results}

Calculations of unsteady cavitating flow were carried out, for four partial flow rates, on the blade cascade of a two-bladed inducer. The unsteady cavitating flow was characterized by the flow coefficient, $\Phi$; the head coefficient, $\Psi$; and the cavitation number, $\sigma$; given by:
$\Phi=\frac{Q}{A_{1} v_{1}}, \quad \Psi=\frac{P_{2}-P_{1}}{(1 / 2) \rho_{l} v_{1}^{2}}, \quad$ and $\quad \sigma=\frac{P_{1}-P_{\text {sat }}}{(1 / 2) \rho_{l} v_{1}^{2}}$

During the performance of this study, various forms and behaviors of cavitation have been observed in the blade cascade. These cavitations are mainly influenced by the flow rate and cavitation number. Therefore, three typical cavitation behaviors, observed in the blade cascade, are described.

\subsubsection{Stable cavitation sheet analysis}

The first numerical results obtained at a flow rate near nominal conditions, $Q=0.97 \cdot Q_{\text {nom, }}$, presented a steady cavitating behavior. The cavitation zone starts to form at the leading edge, on suction side of blade, for high values of $\sigma$. The vapor sheet is symmetrical on both blades all along, for all values of $\sigma$. These cavitation zones increase gradually as $\sigma$ decreases until that the vapor region become large enough to block the flow channel causing the head break down.

When the flow rate decreases to $Q=0.81 \cdot Q_{\text {nom }}$, the cavitation presents a different behavior from that observed when the inducer works at nominal conditions. In partial flows, the alternate blade cavitation occurs during a range of $\sigma$ values. Fig. 5(a) shows the contours of vapor volume fraction for $Q=0.81 \cdot Q_{\text {nom }}$ and different values of $\sigma$. In this figure it is noticed that the cavitation begins with very small vapor zones, which are formed at the leading edge, on suction side of blades. Thus, for values of $\sigma$ between $\sigma=0.723$ and $\sigma=0.219$, symmetrical cavitation sheets attached to each blade can be observed. If $\sigma$ decreases even more, the cavitation zone increases, and it begins to obstruct the flow channel. At $\sigma=0.219$, the length of cavitation sheet, containing $10 \%$ of vapor in volume, is approximately $60 \%$ of the blade spacing, $h$. When $\sigma$ decreases to $\sigma=0.174$ the alternate blade cavitation can be observed on the blade cascade. This asymmetrical cavitation length continued until $\sigma=0.140$. Afterwards, for $\sigma=0.114$, the cavitation sheet becomes symmetrical, therefore the cavitation length are the same on both blades.

Alternate blade cavitation is a phenomenon in which the cavitation length on the blades changes alternately from blade to blade. According to Tsujimoto [25], the alternate blade cavitation starts to develop when the cavitation length, $l_{\text {cav }}$, exceeds about $65 \%$ of the blade spacing. The incidence angle to the neighboring blade decreases and hence the cavitation length on the neighboring blade also decreases. Then, the incidence angle of the original blade increases and thus the cavitation length on it also increases.

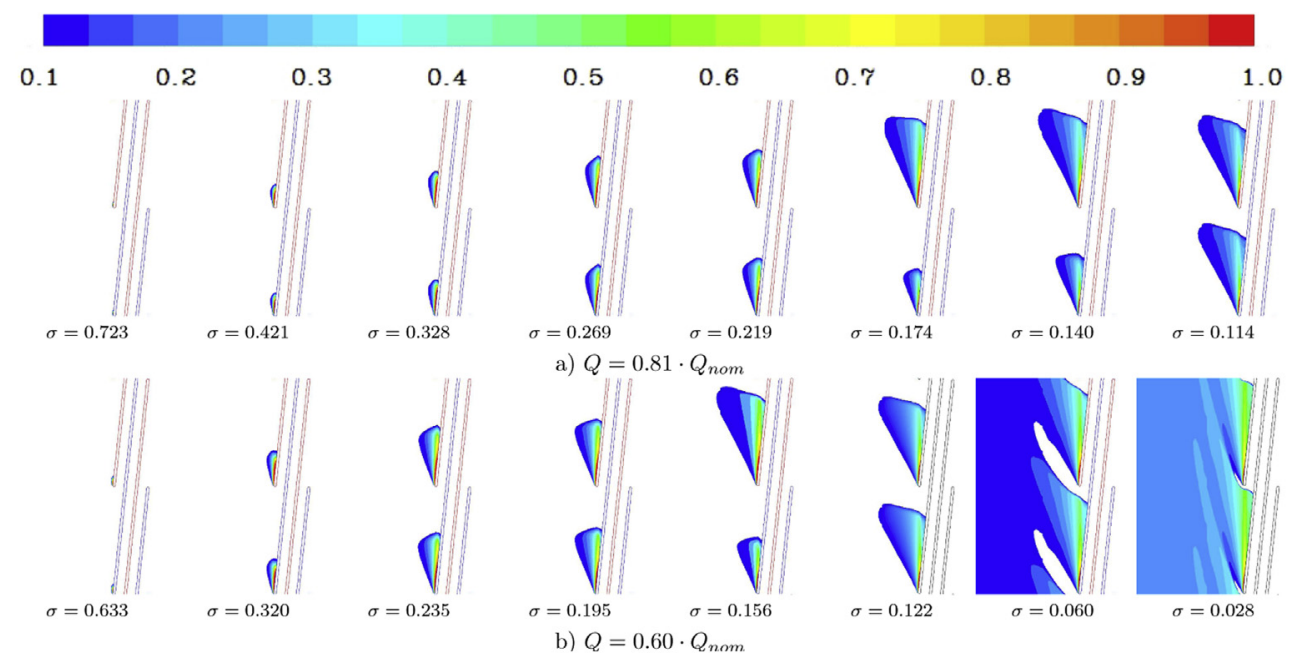

Fig. 5. Alternate blade cavitation ( $\alpha \geq 10 \%)$. 
Fig. 5(b) presents the cavitation sheet behavior for $Q=0.60 \cdot Q_{\text {nom. }}$. Analogous to previous flow analysis, the alternate blade cavitation starts as soon as the $l_{\text {cav }} / h$ ratio is higher than $65 \%$, i.e. for $\sigma=0.156$. Afterward, the cavitation sheet becomes symmetrical for lower values of sigma. These cavitation zones increase gradually as $\sigma$ decreases until the head break down occurs.

\subsubsection{Unstable cavitation sheet analysis}

Numerical simulations in cavitation regime present divergence problems when the flow rate is very small (below $Q=0.55 \cdot Q_{\text {nom }}$ for this case study). The lowest flow rate in which, it was possible to obtain reliable numerical results was $Q=0.56 \cdot Q_{\text {nom. }}$. With this flow rate, the cavitation sheet presented different behaviors. Symmetrical cavitation lengths were observed at high values of $\sigma$, similar to previous cases. However, fluctuations of the cavity length could be observed when $\sigma=0.258$. After that, the cavity length becomes symmetrical on both blades at values of $\sigma$ lower than 0.185 .

Fig. 7(a) shows the contours of vapor volume fraction $(\alpha \geq 10 \%)$ at different points of one rotating cavitation cycle, $T_{\text {cav }}$ Observing the behavior of the cavity length, it is noticed that, at the beginning of the cycle when $t=0.03 \cdot T_{\text {cav }}$, the cavitation lengths are very similar on both blades. The cavitation length gradually decreases over time on blade 1 , while this one increases on blade 2 . Thus, when $t=0.27 \cdot T_{\mathrm{cav}}, l_{\mathrm{cav}}$ is the smallest on blade 1 and the largest on blade 2 . It is clear that, from $t=0.27 \cdot T_{\text {cav }}$ to $t=0.77 \cdot T_{\text {cav }}, l_{\text {cav }}$ decreases gradually on blade 2 , and conditions are reversed being $l_{\text {cav }}$ the smallest on blade 2 and the largest on blade 1 when $t=0.77 \cdot T_{\text {cav }}$.

The temporal evolution of pressure coefficient on leading edge of each blade is shown in Fig. 6, where the reference time, $t_{\text {ref, has }}$ been defined as the time for one impeller revolution, i.e. $t_{\mathrm{ref}}=1 / f_{\omega}$. The sheet cavitation has a cyclical unsteady behavior, with a low frequency of $f_{\text {cav }}=0.07 \cdot f_{\omega}$ on one blade. The fluctuations of cavitation length change the flow dynamics, which cause static pressure variations upstream. The frequency analysis gives, on the absolute reference frame, $f_{\text {cav }}=0.14 \cdot f_{\omega}$ because of the cavitation detachment on both blades.

\subsubsection{Coupling of the instabilities and the self-oscillation of a cavitating sheet}

Numerical simulations were performed using the RNG $\kappa-\varepsilon$ modified turbulence model for a flow rate of $Q=0.56 \cdot Q_{\text {nom }}$ and $\sigma=0.258$. This turbulence model allows the interaction between the unsteadiness of the two blades and the self-oscillation of cavitating sheet.

In this case study, the cavitation sheet presented a similar behavior to the previous analysis, where RNG $\kappa-\varepsilon$ turbulence model was used. The principal difference is that these results show the detachment and convection of the cavitation sheet, as well as the vapor passage from blade 1 to blade 2 . The cavitating sheet fluctuations have a quasi-cyclical behavior with a cavitation detachment frequency of $f_{\text {cav }}=0.12 \cdot f_{\omega}$, in the absolute reference frame.

Fig. 7(b) shows the vapor volume fraction $(\alpha \geq 10)$ at different points of the rotating cavitation cycle, $T_{\text {cav }}$. The vapor sheet detachment on blade 1 can be observed from $t=0.83 \cdot T_{\text {cav }}$ to $t=0.87 \cdot T_{\mathrm{cav}}$, followed by the vapor convection from $t=0.90 \cdot T_{\mathrm{cav}}$ to $t=0.93 \cdot T_{\mathrm{cav}}$, and finally the cavitation passage, through the throat, from blade 1 to blade 2 at $t=1 \cdot T_{\text {cav }}$.

The curves in Fig. 8 show the cavitation length fluctuations ( $\alpha=10 \%)$ for both turbulence models, in where $l_{\text {cav }}$ was obtained directly from Fig. 7.

The dashed lines (RNG $\kappa-\varepsilon$ turbulence model) show a regular fluctuation of the cavities, which remain compacts and adhered to blades. The solid lines (RNG $\kappa-\varepsilon$ modified turbulence model) show an irregular fluctuation of cavity size, which is caused by the detachment and convection of the vapor region. The negative values means that the cavitation sheet grows enough so that a portion of the cavity adheres to the pressure side of the neighbor blade, causing the blockage of this flow channel. Thus, the water flows only through the second flow channel.

The four curves have a similar behavior, but the cavitation lengths are larger when the RNG $k-\varepsilon$ modified model is used. The local length fluctuations observed are caused by the self-oscillation of the cavity.

\subsection{Three-dimensional inducer results}

In a first step, the unsteady cavitating calculations were performed for high cavitation numbers $(\sigma=0.064)$, and from these results, the cavitation number was decreased to observe the flow behavior under different conditions of cavitation ( $\sigma=0.051$ and $\sigma=0.043$ ).

Figs. 9 and 10 show, for $\Phi=0.005$ and $\sigma=0.064$, the isosurface of vapor volume fraction when $\alpha=20 \%$. This value was selected because of it lets us to observe the cavitating fluctuation. In this case, when $\alpha>20 \%$, the isosurface remains constant all along calculations; and when $\alpha<20 \%$, the isosurface hides the fluctuations due to that cavitation is completely developed. The temporal evolution of the cavitation sheet can be observed from $t=126 \cdot t_{\text {ref }}$ to $t=148 \cdot t_{\text {ref }}$. The reference time has been defined as the time for one impeller revolution, i.e. $t_{\mathrm{ref}}=1 / f_{\omega}$. The pictures were obtained when all flow parameters were stabilized, the time step used for the unsteady simulations was $\Delta t=7.5 E-05 \mathrm{~s}$.

In general, the cavitation cloud shows a crown shape on the periphery of the blades, moreover, the cavitation forms a torch upstream of the inducer. The cavitation cloud that is located on the periphery and the cavitation torch are connected by a narrow
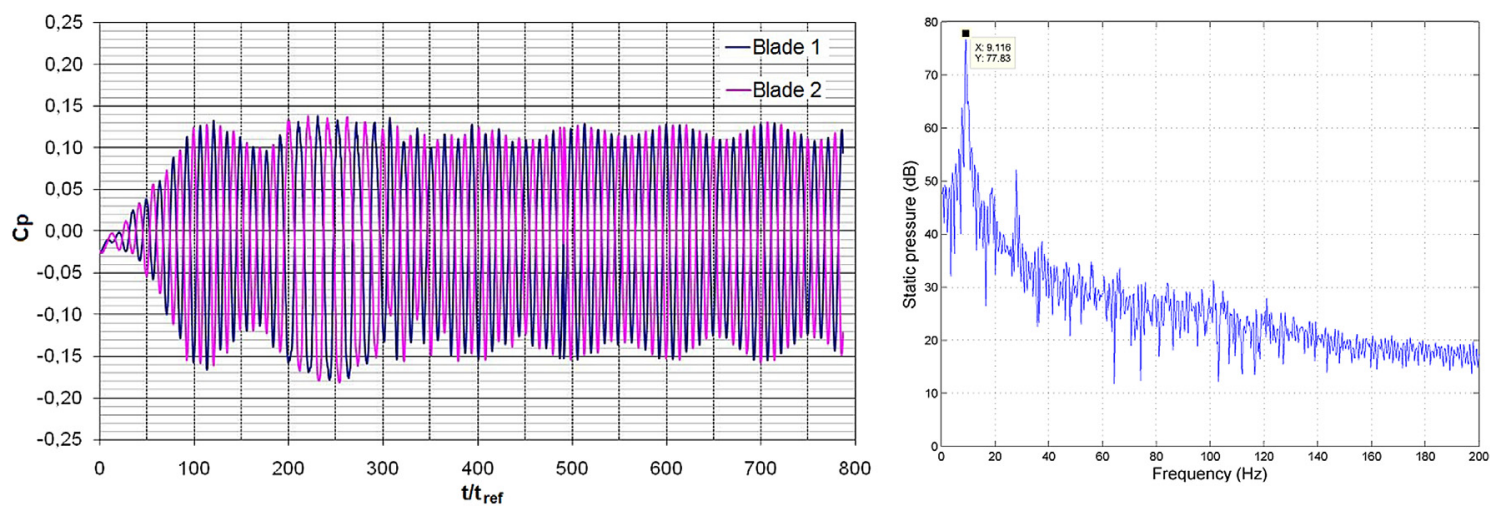

Fig. 6. Temporal evolution of pressure coefficient on the leading edge of blades and the corresponding FFT analysis. 
a) RNG $\kappa-\epsilon$ turbulent model
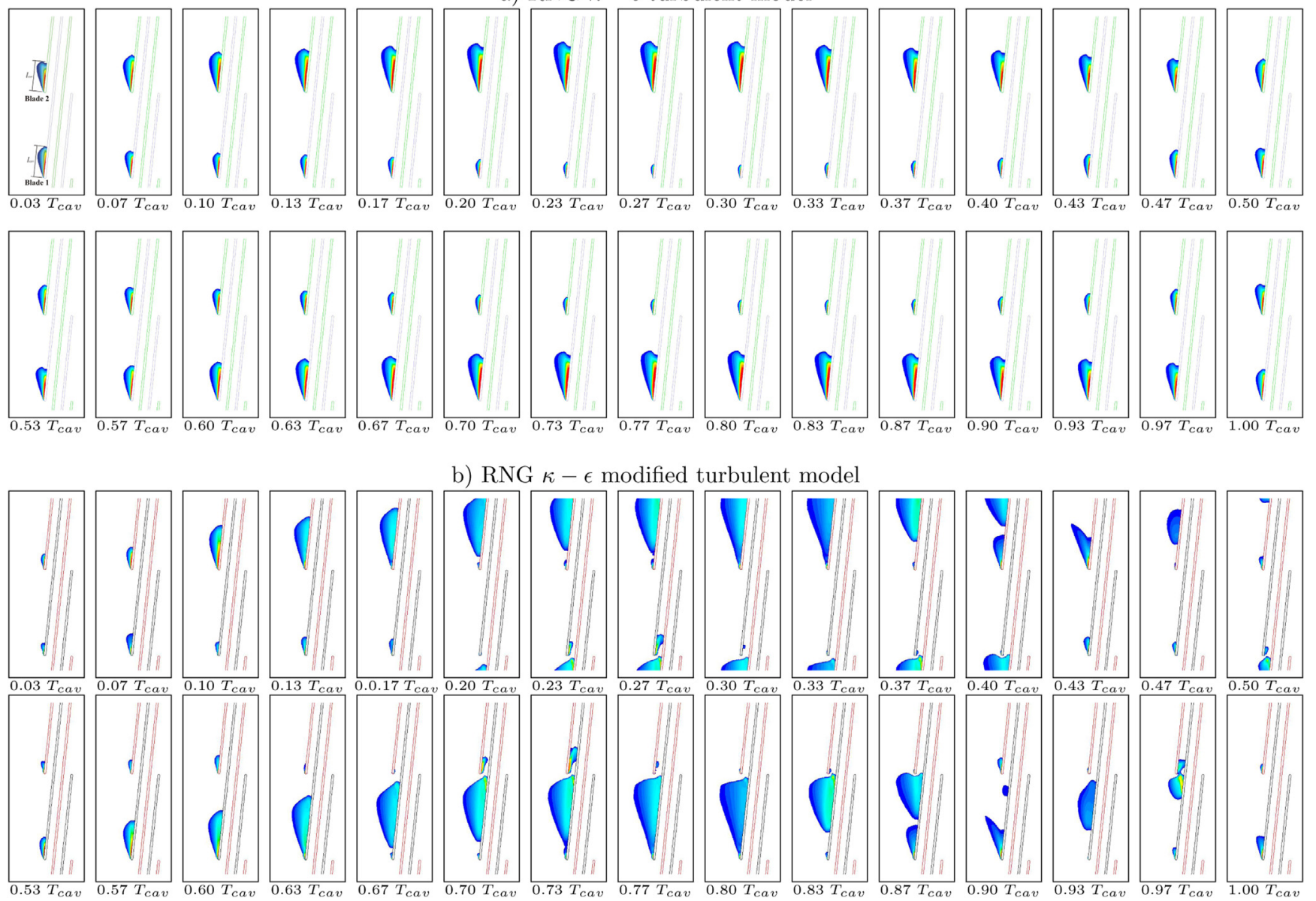

Fig. 7. Rotating cavitation $\left(\alpha \geq 10 \%, \sigma=0.258\right.$, and $\left.Q=0.56 \cdot Q_{\text {nom }}\right)$.

region of vapor formed through the leading edge, from the blade tip to the inducer hub.

Fig. 9 shows that, for $t=126 \cdot t_{\mathrm{ref}}$, the cavitation sheets have similar shapes on both blades. Later, at $t=128 \cdot t_{\mathrm{ref}}$, the cavitation develops gradually on the leading edge of inferior blade (blade 2) until it becomes larger than the cavity on the superior blade (blade 1). Then, the cavitation cloud decreases gradually on the leading edge of blade 2 , until $t=132 \cdot t_{\mathrm{ref}}$, when the cavitation

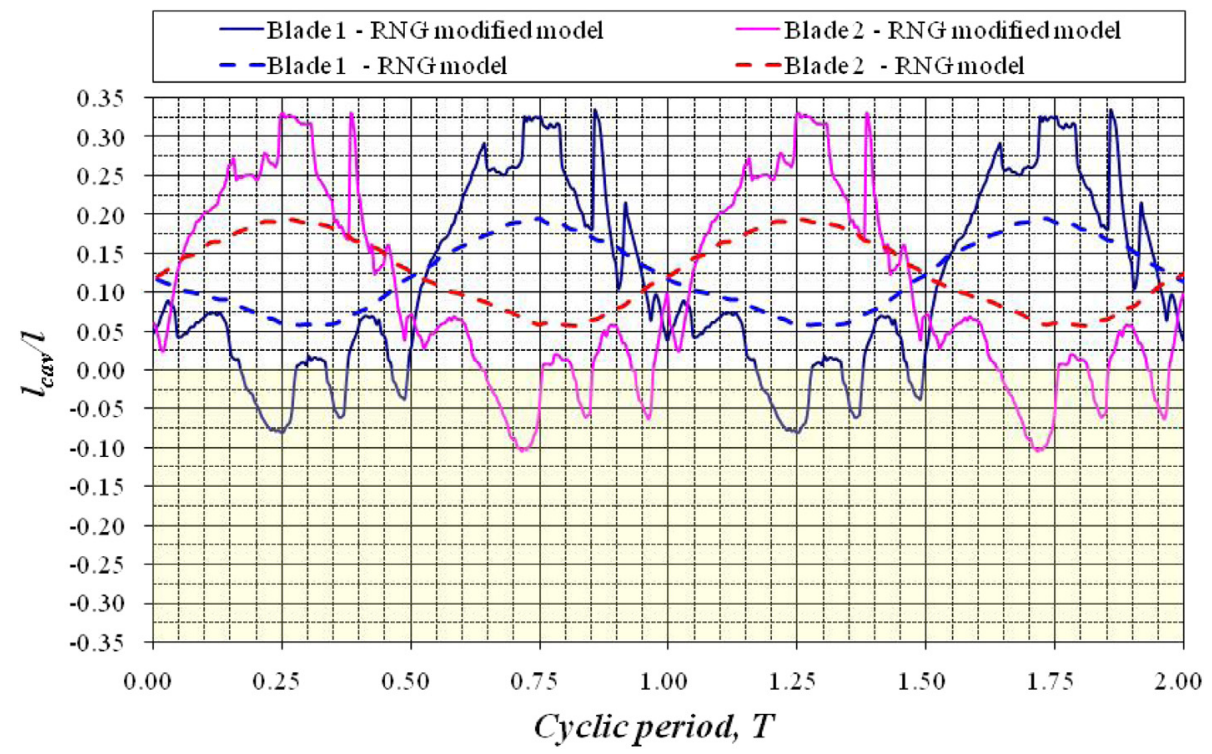

Fig. 8. Comparison of the cavitation lengths calculated by RNG $\kappa-\varepsilon$ and RNG $\kappa-\varepsilon$ modified models. 

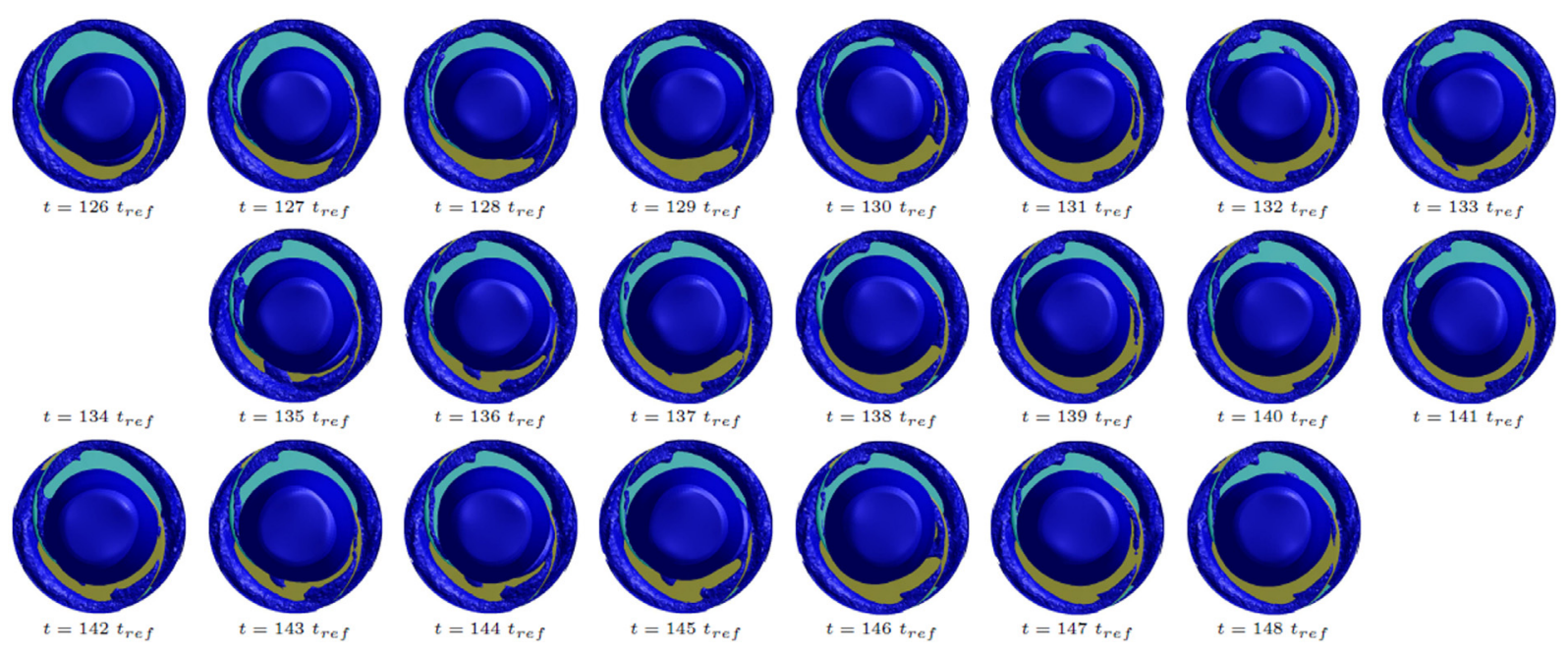

$t=132 t_{r e f}$
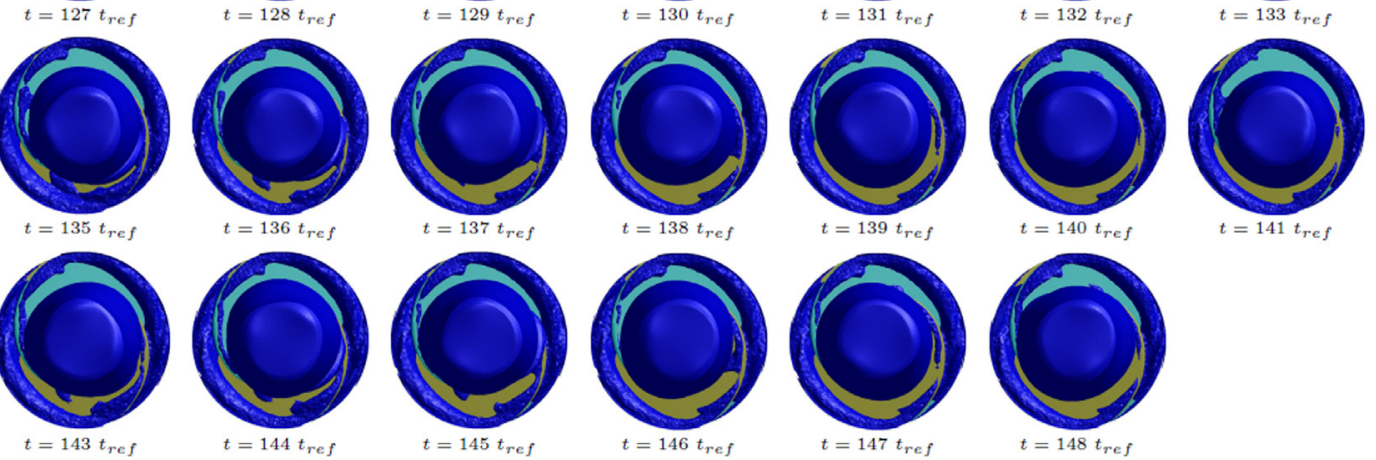

Fig. 9. Temporal evolution of iso-surface of the vapor volume fraction on the inducer, front view $(\alpha=20 \%, \Phi=0.005$ and $\sigma=0.064)$.

cloud is the biggest on blade 1 . Finally, the cavitation cloud begins to grow on blade 2 and it decreases on blade 1, until the cavitation lengths become, at $t=136 \cdot t_{\mathrm{ref}}$, in the biggest and the smallest, respectively.

As can be seen in figures, the fluctuations of the cavitation size have a periodic behavior. The cavitation length is maximal on blade 2 , when $t=128 \cdot t_{\mathrm{ref}} t=136 \cdot t_{\mathrm{ref}}$ and $t=144 \cdot t_{\mathrm{ref}}$. On the other hand, it is maximal on blade 1 , when $t=132 \cdot t_{\mathrm{ref}}, t=140 \cdot t_{\mathrm{ref}}$, and $t=148 \cdot t_{\mathrm{ref}}$. Thus, the cavitation fluctuation period is $T_{\mathrm{cav}}=0.0675 \mathrm{~s}$ and its frequency is $f_{\text {cav }}=14.8 \mathrm{~Hz}$.

Fluctuations can be driven by the cavitation torch formed upstream of the inducer. The torch runs in the direction of the inducer rotation. So, the torch turns one time, while the inducer turns nine times.

On the other hand, numerical results for $\Phi=0.005$ and $\sigma=0.043$ show three successive cycles of the cavitation fluctuation, which occur for 22 inducer cycles. Thus, the cavitation fluctuation period is $T_{\text {cav }}=0.06 \mathrm{~s}$ and its frequency is $f_{\text {cav }}=16.7 \mathrm{~Hz}$.

\section{Conclusions}

Unsteady numerical simulations were carried out over two different configurations: first, in a blade cascade of a two-bladed inducer, then the calculations were realized for a 3D geometry of same inducer.

Cavitating flow in two-bladed inducer, for various $\sigma$ values and flow rates, predicted three types of cavitation behavior on the blade cascade:

1. stable behavior with symmetrical cavitation length,

2. stable behavior with non-symmetrical cavitation length,

3. cyclical unstable behavior with non-symmetrical cavitation length.

Cavitation length behavior was symmetrical and stable for a high flow rate of $Q=0.97 \cdot Q_{\text {nom }}$. Alternate blade cavitation was observed for lower flow rates, when the $l / h$ ratio was higher than about $65 \%$. Finally, the rotating cavitation was observed only for a partial flow rate of $Q=0.56 \cdot Q_{\text {nom }}$, where the calculations were carried out using RNG $\kappa-\varepsilon$ model and RNG $\kappa-\varepsilon$ modified model.

Numerical results showed three different mechanisms of cavitation instabilities:

1. Self-oscillation of the cavitation sheet due to the interaction between the recirculation flow and the cavity surface in the venture geometry.
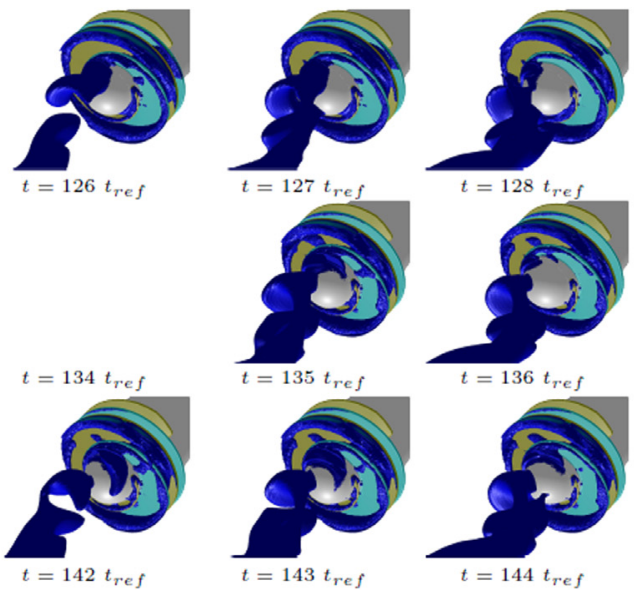

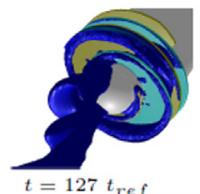

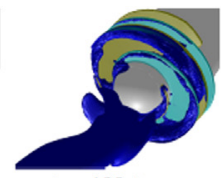
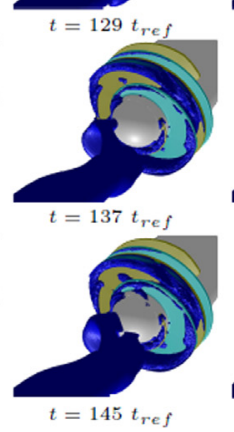
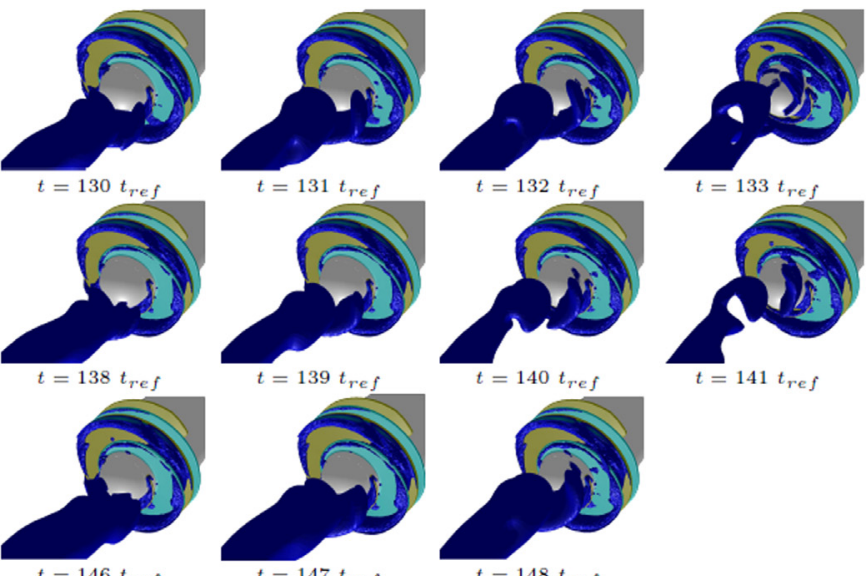

Fig. 10. Temporal evolution of iso-surface of the vapor volume fraction on the inducer, isometric view $(\alpha=20 \%, \Phi=0.005$ and $\sigma=0.064)$. 
2. Rotating cavitation due to the interaction of the sheet cavitation in a blade with the leading edge of the neighbor blade in blade cascade.

3. Coupling of the rotating cavitation and the self-oscillating of the cavitation sheet in blade cascade.

Finally, the unsteady cavitating calculations realized for the three-dimensional inducer have highlighted the difficulty in obtaining numerical results and for compiling and analyzing them. The results show that rotating cavitation appears on 3D geometry but it is less obvious than those on the blade cascade. The shape and behavior of cavitation is greatly disturbed by the radial clearance, which also modifies the torch which is formed upstream of the inducer.

\section{Nomenclature}

$\begin{array}{ll}A & \text { area } \\ D & \text { diameter } \\ f_{\text {cav }} & \text { detachment frequency } \\ f_{\omega} & \text { rotational frequency } \\ h & \text { blade spacing } \\ l_{\text {blade }} & \text { blade chord length } \\ l_{\text {cav }} & \text { cavitation length } \\ P & \text { pressure } \\ p_{v} & \text { vapor pressure } \\ Q & \text { flow rate } \\ R & \text { radius } \\ T_{\omega} & \text { rotational period } \\ T_{\text {cav }} & \text { detachment period } \\ t & \text { turbulent flow } \\ U & \text { tangential velocity } \\ v & \text { velocity magnitude }\end{array}$

Greek

$\alpha \quad$ vapor volume fraction

$\beta \quad$ blade angle

$\gamma \quad$ vapor mass fraction

$\eta \quad$ efficiency

$\rho$ density

$\sigma \quad$ cavitation number

$\sigma_{\mathrm{s}} \quad$ surface tension

$\Phi$ flow coefficient

$\Psi \quad$ head coefficient

$\omega \quad$ rotational speed

\section{Subscript}

1,2 inlet, outlet

a axial direction

B bubble

c condensation

cav cavitation

e vaporization

g gas

$1 \quad$ liquid

nom nominal

tip blade tip

v vapor

$\mathrm{t}$ time

\section{References}

[1] F. Bakir, S. Koudri, R. Noguera, R. Rey, Experimental analysis of an axial inducer influence of the shape of the blade leading edge on the performances in cavitating regime, J. Fluids Eng. 125 (2003) 293-301.

[2] R. Noguera, R. Rey, F. Massouh, F. Bakir, S. Kouidri, Design and analysis of axial pumps, in: ASME Fluids Eng., 2nd Pumping Machinery Symp., 1993, pp. 95-111. Washington DC, USA.

[3] F. Bakir, S. Kouidri, R. Noguera, R. Rey, Design and analysis of axial inducers performances, in: ASME Fluids Eng. Division Summer Meeting, Washington DC, USA, 1998.

[4] Y. Tsujimoto, Y. Yoshida, Y. Maekawa, S. Watanabe, T. Hashimoto, Observation of oscillating cavitation of an inducer, J. Fluids Eng. 119 (1997) 775-781.

[5] A. Cervone, C. Bramanti, L. Torre, D. Fotino, L. d'Agostino, Setup of a highspeed optical system for the characterization of flow instabilities generated by cavitation, J. Fluids Eng. 129 (7) (2007) 877-885.

[6] Y. Yoshida, Y. Sasao, M. Watanabe, T. Hashimoto, Y. Iga, T. Ikohagi, Thermodynamic effect on rotating cavitation in an inducer, J. Fluids Eng. 131 (9) (2009) 0913021-0913027.

[7] Y. Yoshida, H. Nanri, K. Kikuta, Y. Kazami, Y. Iga, T. Ikohagi, Thermodynamic effect on subsynchronous rotating cavitation and surge mode oscillation in a space inducer, J. Fluids Eng. 133 (6) (2011) 0613011-0613017.

[8] J. Franc, G. Boitel, M. Riondet, E. Janson, P. Ramina, C. Rebattet, Thermodynamic effect on a cavitating inducer-part i: geometrical similarity of leading edge cavities and cavitation instabilities, J. Fluids Eng. 132 (2) (2010) 0213031-0213038.

[9] J. Franc, G. Boitel, M. Riondet, E. Janson, P. Ramina, C. Rebattet, Thermodynamic effect on a cavitating inducer-part ii: on-board measurements of temperature depression within leading edge cavities, J. Fluids Eng. 132 (2) (2010) 0213041-0213049.

[10] F. Bakir, R. Rey, A. Gerber, T. Belamri, B. Hutchinson, Numerical and experimental investigations of the cavitating behavior of an inducer, Int. J. Rot. Mach. 10 (1) (2004) 15-25.

[11] I. Mejri, F. Bakir, R. Rey, T. Belamri, Comparison of computational results obtained from a homogenious cavitation model with experimental investigations of three inducers, J. Fluids Eng. 128 (6) (2006) 1308-1323.

[12] R. Campos-Amezcua, S. Khelladi, F. Bakir, Z. Mazur-Czerwiec, C. Sarraf, R. Rey, Numerical analysis of unsteady cavitating flow in an axial inducer, IMechE A J. Power Energy 224 (2) (2010) 223-238.

[13] R. Campos-Amezcua, S. Khelladi, Z. Mazur-Czerwiec, F. Bakir, A. CamposAmezcua, R. Rey, Numerical and experimental study of cavitating flow through an axial inducer considering tip clearance, IMechE A J. Power Energy 227 (8) (2013) 858-868.

[14] N. Tani, N. Yamanishi, Y. Tsujimoto, Influence of flow coefficient and flow structure on rotational cavitation in inducer, J. Fluids Eng. 134 (2) (2012) $0213021-02130213$.

[15] B. Ji, X. Luo, X. Peng, Y. Wu, H. Xu, Numerical analysis of cavitation evolution and excited pressure fluctuation around a propeller in non-uniform wake, Int J. Multiph. Flow. 43 (2012) 13-21.

[16] B. Ji, X. Lou, X. Peng, Y. Wu, Three-dimensional large eddy simulation and vorticity analysis of unsteady cavitating flow around a twisted hydrofoil, J. Hydrodyn. 25 (4) (2013) 510-519.

[17] O. Coutier-Delgosha, R. Fortes-Patella, J. Reboud, Evaluation of the turbulence model influence on the numerical simulation of unsteady cavitation, J. Fluids Eng. 125 (2003) 38-45.

[18] E. Goncalves, Numerical study of unsteady turbulent cavitating flows, Eur. J. Mech. B Fluids 30 (1) (2011) 26-40.

[19] J. Decaix, E. Goncalves, Compressible effects modeling in turbulent cavitating flows, Eur. J. Mech. B Fluids 39 (2013) 11-31.

[20] A. Singhal, M. Athavale, H. Li, Y. Jiang, Mathematical basis and validation of the full cavitation model, J. Fluids Eng. 124 (2002) 617-624.

[21] R. Campos-Amezcua, Analyse des coulements cavitants stationnaires et instationnaires dans les turbomachines, Ph.D. thesis, Arts et Mtiers ParisTech, Paris, France, 2009.

[22] S. Kim, S. Mathur, J. Murthy, D. Chouhury, A reynolds-average navier-stokes solver using unstructured mesh based finite-volume scheme, in: 36th AIAA Aerospace Sciences Meeting and Exhibit, 1998. AIAA 98-0231, Reno, USA.

[23] V. Yakhot, S. Orszag, Renormalization group analysis of turbulence i basic theory, J. Sci. Comput. 1 (1) (1986) 3-51.

[24] J. Reboud, B. Stutz, O. Coutier-Delgosha, Two-phase flow structure of cavitation: Experiment and modeling of unsteady effects, in: 3th Int. Symp. on Cavitation, Grenoble, France, 1998.

[25] Y. Tsujimoto, H. Horiguchi, X. Qiao, Backflow from inducer and its dynamics, in: ASME Fluids Eng. Conf. - 5th Pumping Machinery Symp. Houston, USA, 2005. 